\title{
Time-resolved adaptive FEM simulation of the DLR-F11 aircraft model at high Reynolds number
}

\author{
Johan Hoffman* \\ Computational Technology Laboratory, School of Computer Science and Communication, KTH \\ Johan Jansson ${ }^{\dagger}$ \\ Computational Technology Laboratory, School of Computer Science and Communication, KTH \\ Basque Center for Applied Mathematics, Bilbao, Spain \\ Niclas Jansson ${ }^{\ddagger}$ \\ RIKEN Advanced Institute for Computational Science
}

Rodrigo Vilela de Abreu ${ }^{\S}$

Computational Technology Laboratory, School of Computer Science and Communication, KTH

Linné FLOW Centre, KTH

\begin{abstract}
We present a time-resolved, adaptive finite element method for aerodynamics, together with the results from the HiLiftPW-2 workshop, where this method is used to compute the flow past a DLR-F11 aircraft model at realistic Reynolds number. The mesh is automatically constructed by the method as part of the computation, and no explicit turbulence model is used. The effect of unresolved turbulent boundary layers is modeled by a simple parametrization of the wall shear stress in terms of the skin friction. In the extreme case of very high Reynolds numbers we approximate the small skin friction by zero skin friction, corresponding to a free slip boundary condition, which results in a computational model without any model parameter that needs tuning. Thus, the simulation methodology bypasses the main challenges posed by high Reynolds number CFD: the design of an optimal computational mesh, turbulence (or subgrid) modeling, and the cost of boundary layer resolution. The results from HiLiftPW-2 presented in this report show good agreement with experimental data for a range of different angles of attack, while using orders of magnitude fewer degrees of freedom than what is needed in state of the art methods such as RANS.
\end{abstract}

\section{Introduction}

In this paper, we present computational results obtained for the $2^{\text {nd }}$ AIAA CFD High-Lift Prediction Workshop (HiLiftPW-2), realized in San Diego, California, in 2013, of the flow past a complex geometry, high-lift aircraft model (DLR-F11), see Figure 1. One of the main objectives of the workshop was to "assess numerical prediction capability" 11 of current Computational Fluid Dynamics (CFD) codes and methods.

This is, of course, the main goal of similar past and ongoing workshop efforts, such as the BANC workshop series for the aeroacoustics community. ${ }^{[2}$ The objective of our participation in the HiLiftPW-2 is also connected to this "benchmarking" aspect of such workshops, and is, in this particular regard, in line

\footnotetext{
*Professor, Computational Technology Laboratory, School of Computer Science and Communication, KTH, SE-10044 Stockholm, Sweden.

${ }^{\dagger}$ Research line leader, Computational Technology Laboratory, CSC, KTH, SE-10044 Stockholm, Sweden and Basque Center for Applied Mathematics, Bilbao, Spain.

$¥$ Postdoctoral Researcher, RIKEN Advanced Institute for Computational Science, Chuo-ku, Kobe, 650-0047, Japan

$\S$ PhD candidate, Computational Technology Laboratory, School of Computer Science and Communication, KTH, SE-10044 Stockholm, Sweden.
} 
with the objectives of the other workshop participants: we want to compare our computational results with experiments. We believe these comparisons are, per se, of great scientific value.

However, we have other, long-term goals with our participation, which are perhaps more ambitious, and certainly more difficult to achieve: we would like to present a new methodology for computational aerodynamics, and we would like this methodology to establish a new direction for the field.

The basis for our new methodology is an adaptive finite element method without boundary layer resolution. The mesh is automatically constructed by the method as part of the computation, and no explicit turbulence model is needed. The effect of unresolved turbulent boundary layers is modeled by a simple parametrization of wall shear stress in terms of the skin friction. In the extreme case of very high Reynolds numbers $(R e)$ we approximate the small skin friction by zero skin friction, corresponding to a free slip boundary condition, which results in a computational model without any model parameters that need tuning. Thus, the simulation methodology bypasses the main challenges posed by high Re CFD: the design of an optimal computational mesh, turbulence (or subgrid) modeling, and the cost of boundary layer resolution.

In this paper we present the main components of the simulation methodology and our results from the HiLiftPW-2 workshop, where we highlight the non-standard aspects of the methodology and discuss the results in relation to the experiments. We find that the simulation results compare well with experimental data for all angles of attack, while using orders of magnitude less degrees of freedom than other participants of the workshop. The low computational cost also allows for a time-resolved simulation, which provides additional results that cannot be obtained from a stationary simulation, such as the ones based on Reynoldsaveraged Navier-Stokes equations (RANS).

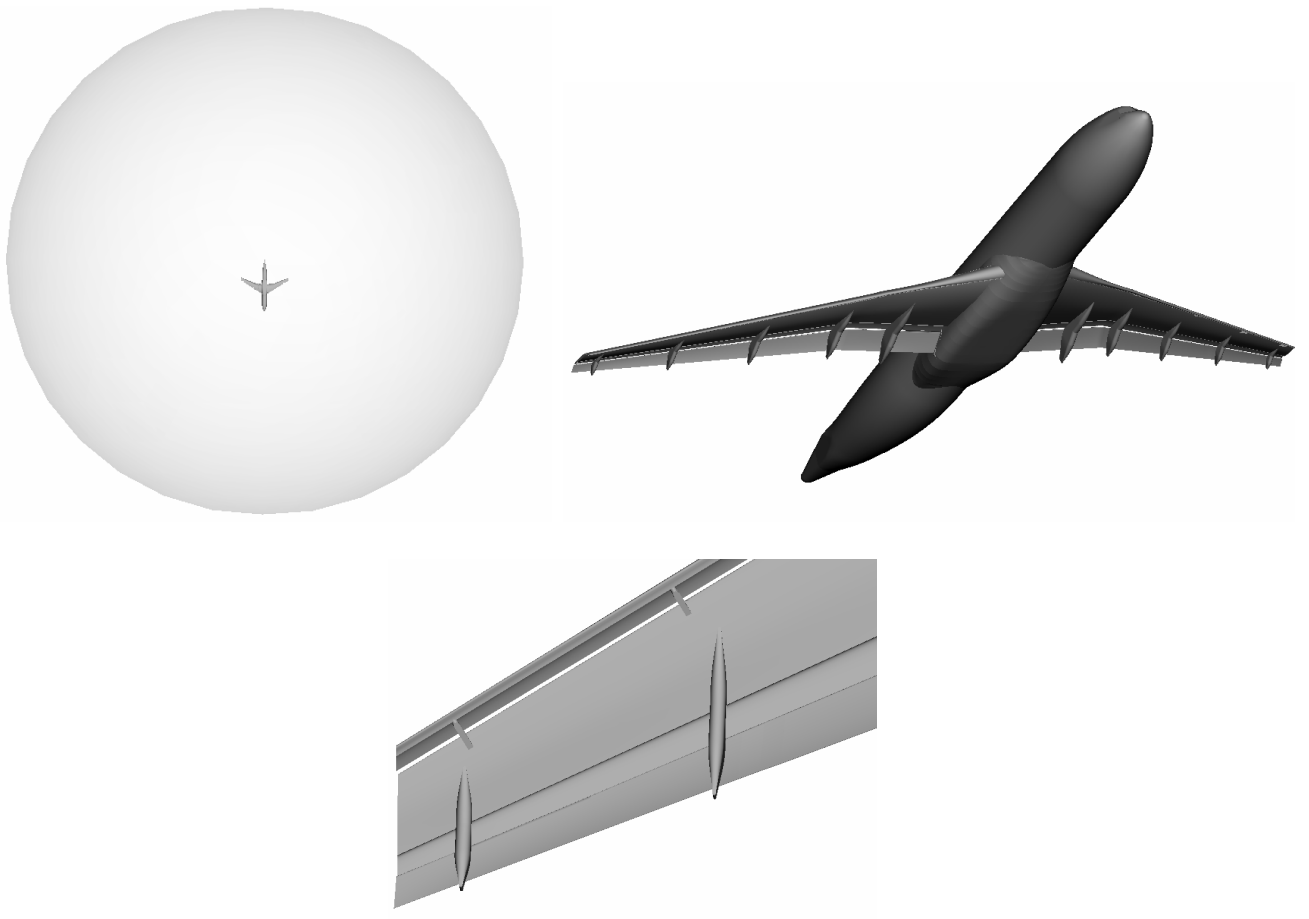

Figure 1. Overview of the domain and of the DLR-F11 aircraft model (upper) and detail of wing pressure side (lower). On the detail snapshot of the wing pressure side, slat tracks and flap fairings are seen.

\section{Simulation Methodology}

The mathematical framework for the simulation method is functional analysis and the concept of weak solutions to the Navier-Stokes equations (NSE), introduced by the mathematician Jean Leray in 1934. Leray proved that there exist weak solutions (or turbulent solutions in the terminology of Leray) that satisfy NSE in variational form, that is NSE integrated against a family of test functions. A finite element method (FEM) is based on the variational form of NSE, and one can show that, if the formulation of the method satisfies 
certain conditions on stability and consistency, the approximate FEM solutions converge towards a weak solution of the NSE as the finite element mesh is refined ${ }^{3}$ We refer to such FEM as General Galerkin (G2) methods.

The test functions in G2 are defined over the mesh, and thus the finest scales of a G2 approximation are set by the mesh size. In contrast to RANS or LES (Large eddy simulation), no averaging operator or filter is applied to NSE, and thus no Reynolds or subgrid stresses that need modeling are introduced. Dissipation of turbulent kinetic energy in under-resolved parts of the flow is provided by the numerical stabilization of G2 in the form of a weighted least squares method based on the residual of NSE. Thus, the method is purely based on the NSE mathematical model, and no other modeling assumptions are made.

In G2, the mesh is adaptively constructed based on a posteriori estimation of the error in chosen goal or target functionals, such as drag and lift forces for example. Using duality in a variational framework, a posteriori error estimates can be derived in terms of the residual, the mesh size, and the solution of an adjoint problem. ${ }^{3}$ We initiate the adaptive mesh refinement algorithm from a coarse mesh, fine enough to capture the geometry, but without any further assumptions on the solution (i.e., no boundary layer meshes or ad hoc mesh design based on expected separation and wake structures are needed). To model the effect of unresolved turbulent boundary layers we use a simple parametrization of the wall shear stress in terms of the skin friction. In particular, for very high $R e$ we approximate the small skin friction by zero skin friction, which corresponds to a free slip boundary condition without boundary layer resolution.

This methodology is validated for a number of standard benchmark problems in the literature, $\frac{4}{5}\left[\begin{array}{l}6 / 7 \\ 7\end{array}\right.$ and in the following sections we describe the basic elements of the G2 method, also referred to as Adaptive DNS/LES, or simply Direct finite element simulation (DFS).

For the particular problem proposed in the HiLiftPW-2, we used a low order finite element discretization on unstructured tetrahedral meshes, which we refer to as $\mathrm{cG}(1) \mathrm{cG}(1)$, i.e., continuous piecewise linear approximations in space and time.

\section{A. The cG(1)cG(1) method}

As the basic model for incompressible Newtonian fluid flow, we consider the NSE with constant kinematic viscosity $\nu>0$, enclosed in $\Omega \subset \mathbb{R}^{3}$, with boundary $\Gamma$, over a time interval $I=(0, T]$ :

$$
\begin{aligned}
& \dot{u}+(u \cdot \nabla) u+\nabla p-2 \nu \nabla \cdot \epsilon(u)=f, \quad(x, t) \in \Omega \times I, \\
& \nabla \cdot u=0, \quad(x, t) \in \Omega \times I, \\
& u(x, 0)=u^{0}(x), \quad x \in \Omega,
\end{aligned}
$$

with $u(x, t)$ the velocity vector, $p(x, t)$ the pressure, $u^{0}(x)$ initial data and $f(x, t)$ a body force. Moreover, $\sigma_{i j}=-\nu \epsilon_{i j}(u)+p \delta_{i j}$ is the stress tensor, with the strain rate tensor $\epsilon_{i j}(u)=1 / 2\left(\partial u_{i} / \partial x_{j}+\partial u_{j} / \partial x_{i}\right)$, and $\delta_{i j}$ the Kronecker delta function. The relative importance of viscous and inertial effects in the flow is determined by the Reynolds number $R e=U L / \nu$, where $U$ and $L$ are characteristic velocity and length scales.

The $\mathrm{cG}(1) \mathrm{cG}(1)$ method is based on the continuous Galerkin method $\mathrm{cG}(1)$ in space and time. With $\mathrm{cG}(1)$ in time, the trial functions are continuous, piecewise linear and the test functions piecewise constant. $\mathrm{cG}(1)$ in space corresponds to both test functions and trial functions being continuous, piecewise linear. Let $0=t_{0}<t_{1}<\ldots<t_{N}=T$ be a sequence of discrete time steps, with associated time intervals $I_{n}=\left(t_{n-1}, t_{n}\right)$ of length $k_{n}=t_{n}-t_{n-1}$, and let $W \subset H^{1}(\Omega)$ be a finite element space consisting of continuous, piecewise linear functions on a tetrahedral mesh $\mathcal{T}=\{K\}$ of mesh size $h(x)$, with $W_{w}$ the functions $v \in W$ satisfying the Dirichlet boundary condition $\left.v\right|_{\Gamma}=w$.

We seek $\hat{U}=(U, P)$, continuous piecewise linear in space and time, and the $\mathrm{cG}(1) \mathrm{cG}(1)$ method for NSE with homogeneous Dirichlet boundary conditions reads: for $n=1, \ldots, N$, find $\left(U^{n}, P^{n}\right) \equiv\left(U\left(t_{n}\right), P\left(t_{n}\right)\right)$, with $U^{n} \in V_{0} \equiv\left[W_{0}\right]^{3}$ and $P^{n} \in W$, such that:

$$
\begin{aligned}
& \left(\left(U^{n}-U^{n-1}\right) k_{n}^{-1}+\bar{U}^{n} \cdot \nabla \bar{U}^{n}, v\right)+\left(2 \nu \epsilon\left(\bar{U}^{n}\right), \epsilon(v)\right)-\left(P^{n}, \nabla \cdot v\right) \\
& +\left(\nabla \cdot \bar{U}^{n}, q\right)+S D_{\delta}^{n}\left(\bar{U}^{n}, P^{n} ; v, q\right)=(f, v), \quad \forall \hat{v}=(v, q) \in V_{0} \times W,
\end{aligned}
$$

where $\bar{U}^{n}=\frac{1}{2}\left(U^{n}+U^{n-1}\right)$ is piecewise constant in time over $I_{n}$, with the stabilizing term

$$
\begin{aligned}
& S D_{\delta}^{n}\left(\bar{U}^{n}, P^{n} ; v, q\right) \equiv \\
& \quad\left(\delta_{1}\left(\bar{U}^{n} \cdot \nabla \bar{U}^{n}+\nabla P^{n}-f\right), \bar{U}^{n} \cdot \nabla v+\nabla q\right)+\left(\delta_{2} \nabla \cdot \bar{U}^{n}, \nabla \cdot v\right),
\end{aligned}
$$


and

$$
\begin{aligned}
(v, w) & =\sum_{K \in \mathcal{T}} \int_{K} v \cdot w d x, \\
(\epsilon(v), \epsilon(w)) & =\sum_{i, j=1}^{3}\left(\epsilon_{i j}(v), \epsilon_{i j}(w)\right),
\end{aligned}
$$

with the stabilization parameters $\delta_{1}=\kappa_{1}\left(k_{n}^{-2}+\left|U^{n-1}\right|{ }^{2} h^{-2}\right)^{-1 / 2}$ and $\delta_{2}=\kappa_{2} h\left|U^{n-1}\right|$, where $\kappa_{1}$ and $\kappa_{2}$ are positive constants of unit size. We choose a time step size $k_{n}=C_{C F L} \min _{x \in \Omega} h /\left|U^{n-1}\right|$, with $C_{C F L}$ typically in the range $[0.5,20]$. The resulting non-linear algebraic equation system is solved with a robust Schur-type fixed-point iteration method.

\section{B. The Adaptive Algorithm}

A simple description of the adaptive algorithm, starting from $k=0$, reads:

1. For the mesh $\mathcal{T}_{k}$ : compute primal and (linearized) adjoint problem.

2. If $\sum_{K \in \mathcal{T}_{k}} \mathcal{E}_{K}<T O L$ then stop, else:

3. Mark $10 \%$ of the elements with highest $\mathcal{E}_{K}$ for refinement.

4. Generate the refined mesh $\mathcal{T}_{k+1}$, and goto 1 .

Here, $\mathcal{E}_{K}$ is the error indicator for each cell $K$, which we describe in Section C For now, it suffices to say that $\mathcal{E}_{K}$ is a function of the residual of the NSE and of the solution of a linearized adjoint problem. The formulation of the adjoint problem includes the definition of a target functional for the refinement, which usually enters the adjoint equations as a boundary condition or as a volume source term. This functional should be chosen according to the problem we are solving. In other words, one needs to ask the right question in order to obtain the correct answer from the algorithm. In this paper our target functional is chosen to be the mean value in time of the aerodynamic forces.

Apart from a suitable formulation of the adjoint problem, the only other input required from the user is an initial discretization of the geometry, $\mathcal{T}_{0}$. Since our method is designed for tetrahedral meshes that do not require any special treatment of the near wall region (no need for a boundary-layer mesh), the initial mesh can be easily created with any standard mesh generation tool.

\section{A posteriori error estimate for cG(1)cG(1)}

The a posteriori error estimate is based on the following theorem (for a detailed proof, see Chapter 30 here $^{3}$ ):

Theorem 1 If $\hat{U}=(U, P)$ solves (2), $\hat{u}=(u, p)$ is a weak NSE solution, and $\hat{\varphi}=(\varphi, \theta)$ solves an associated adjoint problem with data $M(\cdot)$, then we have the following a posteriori error estimate for the target functional $M(\hat{U})$ with respect to the reference functional $M(\hat{u})$ :

$$
\begin{aligned}
& |M(\hat{u})-M(\hat{U})| \leq \sum_{n=1}^{N}\left[\int_{I_{n}} \sum_{K \in \mathcal{T}_{n}}\left|R_{1}(\hat{U})\right|_{K} \cdot \omega_{1} d t\right. \\
& \left.+\int_{I_{n}} \sum_{K \in \mathcal{T}_{n}}\left|R_{2}(U)\right|_{K} \omega_{2} d t+\int_{I_{n}} \sum_{K \in \mathcal{T}_{n}}\left|S D_{\delta}^{n}(\hat{U} ; \hat{\varphi})_{K}\right| d t\right]=: \sum_{K \in \mathcal{T}_{n}} \mathcal{E}_{K}
\end{aligned}
$$

with

$$
\begin{aligned}
& R_{1}(\hat{U})=\dot{U}+(U \cdot \nabla) U+\nabla P-2 \nu \nabla \cdot \epsilon(u)-f, \\
& R_{2}(U)=\nabla \cdot U
\end{aligned}
$$

where $S D_{\delta}^{n}(\cdot ; \cdot)_{K}$ is a local version of the stabilization form (3), and the stability weights are given by

$$
\begin{aligned}
& \omega_{1}=C_{1} h_{K}|\nabla \varphi|_{K}, \\
& \omega_{2}=C_{2} h_{K}|\nabla \theta|_{K},
\end{aligned}
$$


where $h_{K}$ is the diameter of element $K$ in the mesh $\mathcal{T}_{k}$, and $C_{1,2}$ represent interpolation constants. Moreover, $|w|_{K} \equiv\left(\left\|w_{1}\right\|_{K},\left\|w_{2}\right\|_{K},\left\|w_{3}\right\|_{K}\right)$, with $\|w\|_{K}=(w, w)_{K}^{1 / 2}$, and the dot denotes the scalar product in $\mathbb{R}^{3}$.

For simplicity, it is here assumed that the time derivatives of the dual variables $\hat{\phi}=(\phi, \theta)$ can be bounded by their spatial derivatives. Given Theorem 1 1 , we can understand the adaptive algorithm. As mentioned above, the error indicator, $\mathcal{E}_{K}$, is a function of the residual of the NSE and of (the gradients of) the solution of a linearized adjoint problem (a detailed formulation of the adjoint problem is given in Chapter 14 of Hoffman and Johnsor ${ }^{3}$ ). Thus, on a given mesh, we must first solve the NSE to compute the residuals, $R_{1}(\hat{U})$ and $R_{2}(U)$, and then a linearized adjoint problem to compute the weights multiplying the residuals, $\omega_{1}$ and $\omega_{2}$. With that information, we are able to compute $\sum_{K \in \mathcal{T}_{k}} \mathcal{E}_{K}$ and check it against the given stop criterion. This procedure of solving the forward and backward problems for the NSE is closely related to an optimization loop and can be understood as the problem of finding the "optimal mesh" for a given geometry and boundary conditions, i.e., the mesh with the least possible number of degrees of freedom for computing $M(\hat{u})$ within a given degree of accuracy.

\section{Turbulent boundary layers}

In our work on high Reynolds number turbulent flow,, 9$] 10[11$ we have chosen to apply a skin friction stress as wall layer model. That is, we append the NSE with the following boundary conditions:

$$
\begin{aligned}
& u \cdot n=0, \\
& \beta u \cdot \tau_{k}+n^{T} \sigma \tau_{k}=0, \quad k=1,2,
\end{aligned}
$$

for $(x, t) \in \Gamma_{\text {solid }} \times I$, with $n=n(x)$ an outward unit normal vector, and $\tau_{k}=\tau_{k}(x)$ orthogonal unit tangent vectors of the solid boundary $\Gamma_{\text {solid }}$. We use matrix notation with all vectors $v$ being column vectors and the corresponding row vector is denoted $v^{T}$.

With skin friction boundary conditions, the rate of kinetic energy dissipation in $\mathrm{cG}(1) \mathrm{cG}(1)$ has a contribution of the form:

$$
\sum_{k=1}^{2} \int_{0}^{T} \int_{\Gamma_{\text {solid }}}\left|\beta^{1 / 2} \bar{U} \cdot \tau_{k}\right|^{2} d s d t
$$

from the kinetic energy which is dissipated as friction in the boundary layer. For high Re, we model $R e \rightarrow \infty$ by $\beta \rightarrow 0$, so that the dissipative effect of the boundary layer vanishes with large Re. In particular, we have found that a small $\beta$ does not influence the solution. 9 For the present simulations we used the approximation $\beta=0$, which can be expected to be a good approximation for real high-lift configurations, where $R e$ is typically much larger than in a wind tunnel experiment.

\section{Results}

This section is divided in four parts: in the first and second ones, a detailed comparison of the computed aerodynamic force and pressure coefficients with their corresponding experimental values is presented. In the third part, different flow visualizations are presented and discussed. Here, we would like to make it clear that the results shown in Sections A through C were all obtained with the geometry designated as "config 4 " by the organizing committee ${ }^{1}$ which includes "slat tracks" and "flap track fairings", but no "pressure tube bundles". Finally, we close the section with preliminary results for the geometry including pressure tube bundles, which is the one designated as "config 5 " by the organizing committee.

\section{A. Aerodynamic Forces}

As discussed in Section [II, our simulation methodology is an iterative process, where the mesh is refined in each iteration based on an a posteriori error estimate. Therefore, we could say that the classical mesh study - an essential part of any thoroughly conducted CFD analysis - is a "by-product" of our method, i.e., the method automatically requires the computation of the aerodynamic forces on a series of successively refined meshes.

Figure 2(a) shows the lift coefficient, $C_{L}$, as a function of the angle of attack, $\alpha$, for the finest mesh obtained for each angle. The approach we chose was, starting from the same coarse mesh, to execute the 
adaptive algorithm for each angle of attack separately, resulting in a different "family of meshes" for each angle

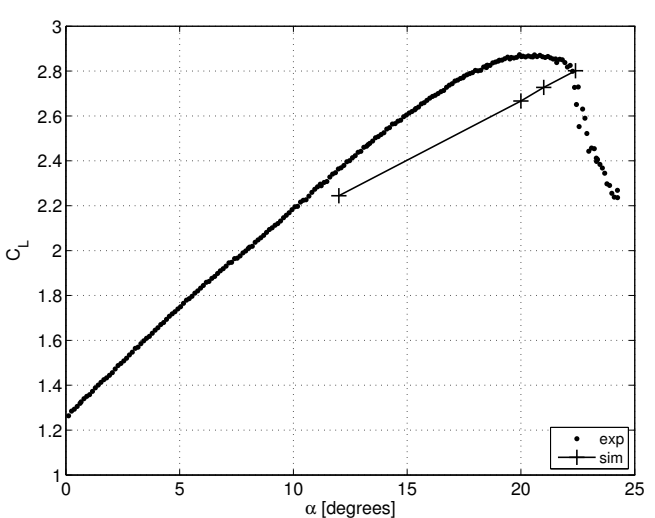

(a) Lift vs. $\alpha$, last iteration (finest mesh).

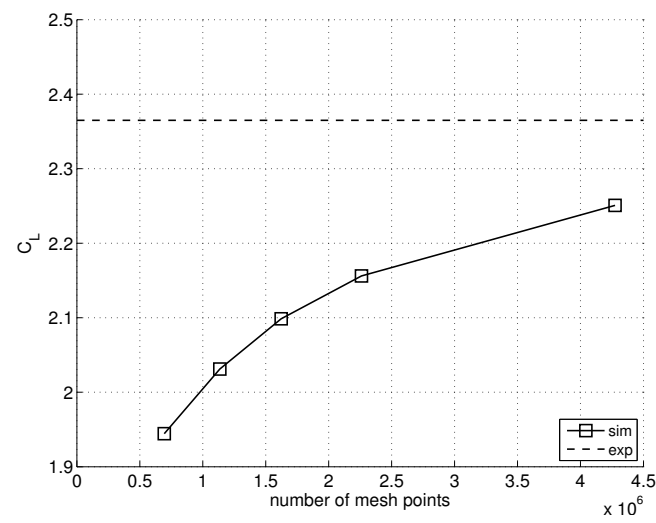

(c) Lift vs. number of mesh points, $\alpha=12^{\circ}$.

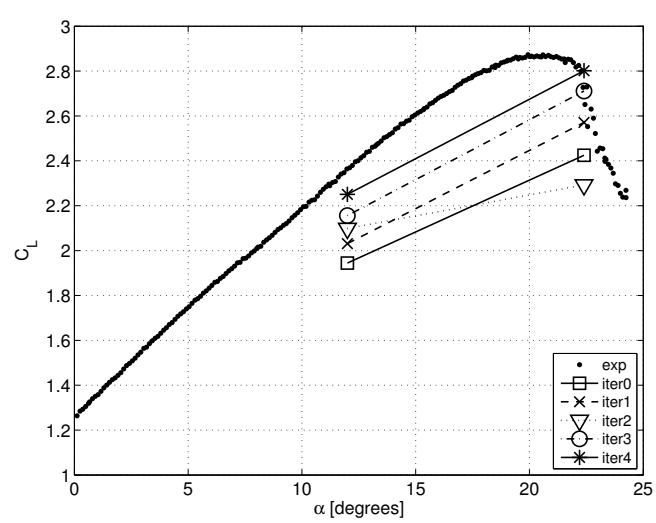

(b) Lift vs. $\alpha$, all iterations.

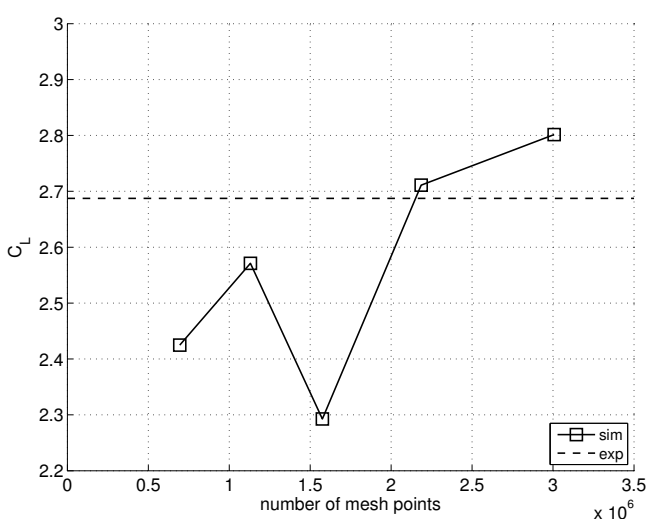

(d) Lift vs. number of mesh points, $\alpha=22.4^{\circ}$.

Figure 2. Lift coefficient, $C_{L}$, vs. angle of attack, $\alpha$, and vs. number of mesh points.

Convergence results are shown in Figure 2(b), where $C_{L}$ is again plotted as a function of $\alpha$, this time for all five iterations of the adaptive algorithms for $\alpha=12^{\circ}, 22.4^{\circ}$. Although no definite convergence can be observed, neither for $\alpha=12^{\circ}$ nor $\alpha=22.4^{\circ}$, we clearly see that the computational result "approaches" the experimental curve as the number of iterations (or points in the mesh) increases. Figures 2(c)] and 2(d) reinforce this trend, showing how $C_{L}$ varies with the number of mesh points, approaching the experimental value for both angles. Similar results for the drag coefficient are shown in Figures 3

Comparisons with experimental values of both $C_{L}$ and $C_{D}$ are shown in Table 1. For $\alpha=12^{\circ}$, we obtained $C_{L}=2.24$ with our finest mesh, which corresponds to a relative error of $5.1 \%$, when compared to the experimental value. For $\alpha=22.4^{\circ}$, the relative error in lift coefficient amounts to $4.2 \%$.

Also shown in the table is a comparison of the lift to drag ratio of our simulation estimates with corresponding wind-tunnel values. The results here are of mixed quality: whereas for $\alpha=12^{\circ}$ the error is less than $5 \%$, the error for $\alpha=22.4^{\circ}$ surpasses $20 \%$.

The timestep $k$ is chosen to be proportional to the mesh size, $h$, according to $k_{n}=C_{C F L} \min _{K \in \mathcal{T}_{k}} h /\left|U^{n-1}\right|$, and thus refining $h$ implicitly also refines $k$. Since $k$ is given by a minimum of $h$ divided by $U$, the timestep may be constrained by small mesh cells determined by detailed geometrical features, which may not have a significant effect on the average global aerodynamic quantities. To quantify this, we performed computations on the coarsest mesh of "config 5", for an angle of attack $\alpha=22.4$, using 3 different timesteps given by $C_{C F L}=4,8,16$. The corresponding $C_{L^{-}}$and $C_{D^{-}}$values vary within $\leq 1 \%$, giving an indication that these quantities are not sensitive to the timestep size in this range. Additionally, the additional cost in average

\footnotetext{
${ }^{a}$ However, due to lack of time, we were only able to obtain adaptive iterations for $\alpha=12^{\circ}, 22.4^{\circ}$; the results for the remaining angles in the figure were computed with the mesh for $\alpha=22.4^{\circ}$.
} 


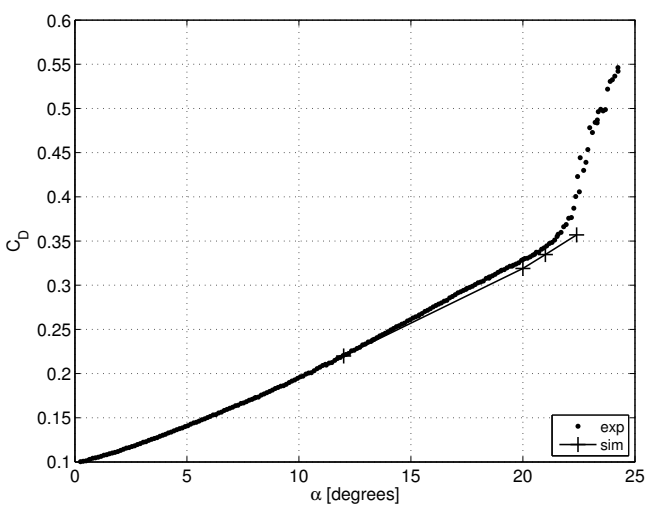

(a) Drag, last iteration (finest mesh).

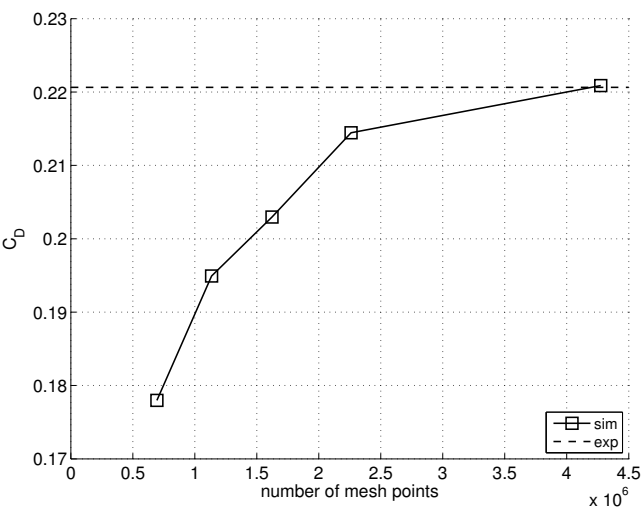

(c) Drag vs. number of mesh points, $\alpha=12^{\circ}$.

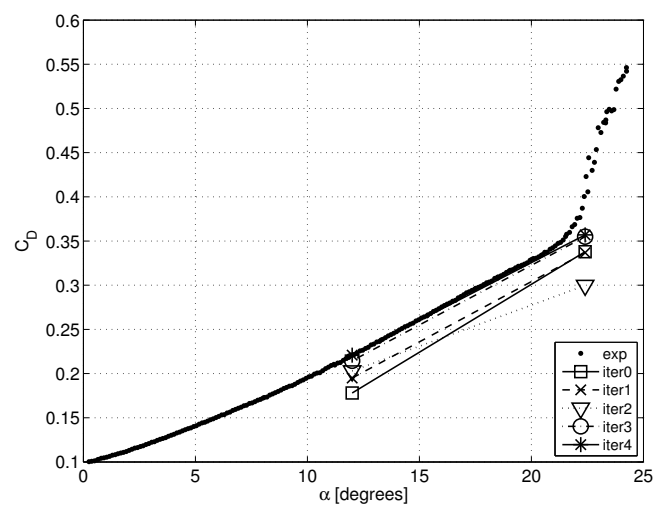

(b) Drag, all iterations.

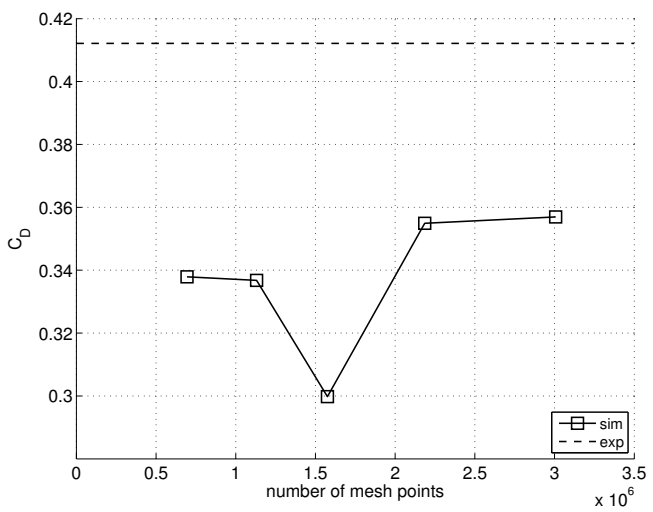

(d) Drag vs. number of mesh points, $\alpha=22.4^{\circ}$.

Figure 3. Drag coefficient, $C_{D}$, vs. angle of attack, $\alpha$, and vs. number of mesh points.

wall-clock time per timestep is $24 \%$ for $C_{C F L}=16$ compared to $C_{C F L}=8$ and $7 \%$ for $C_{C F L}=8$ compared to $C_{C F L}=4$, indicating a significant saving of computational cost when using the larger timesteps in the range.

(a) $\alpha=12^{\circ}$.

\begin{tabular}{|l|c|c|c|}
\hline & sim. & exp. & relative error \\
\hline lift & 2.24 & 2.36 & $5.1 \%$ \\
drag & 0.220 & 0.221 & $<1 \%$ \\
ratio & 10.2 & 10.7 & $4.8 \%$ \\
\hline
\end{tabular}

(b) $\alpha=22.4^{\circ}$.

\begin{tabular}{|l|c|c|c|}
\hline & sim. & exp. & relative error \\
\hline lift & 2.80 & 2.69 & $4.2 \%$ \\
drag & 0.357 & 0.412 & $13.4 \%$ \\
ratio & 7.85 & 6.52 & $20.3 \%$ \\
\hline
\end{tabular}

Table 1. Comparison of drag and lift coefficients and lift over drag ratio, $L / D$, against experiments.

\section{B. Pressure coefficients}

Mean pressure coefficients, $C_{P}$, were measured at different distances from the aircraft body along the wing in order to verify how lift is generated over the wing surface and how and where it breaks down after stall. Here, we present $C_{P}$ distributions for selected measurement stations and for the angles of attack for which we have performed adaptive iterations, i.e. $\alpha=12^{\circ}, 22.4^{\circ}$.

Figure 4 shows $C_{P}$ at four measurement locations $\mathrm{b}^{\mathrm{b}}$ for $\alpha=12^{\circ}$, one close to the aircraft body, $P S 01$, one

\footnotetext{
${ }^{\mathrm{b}}$ Omitted locations show similar trends.
} 

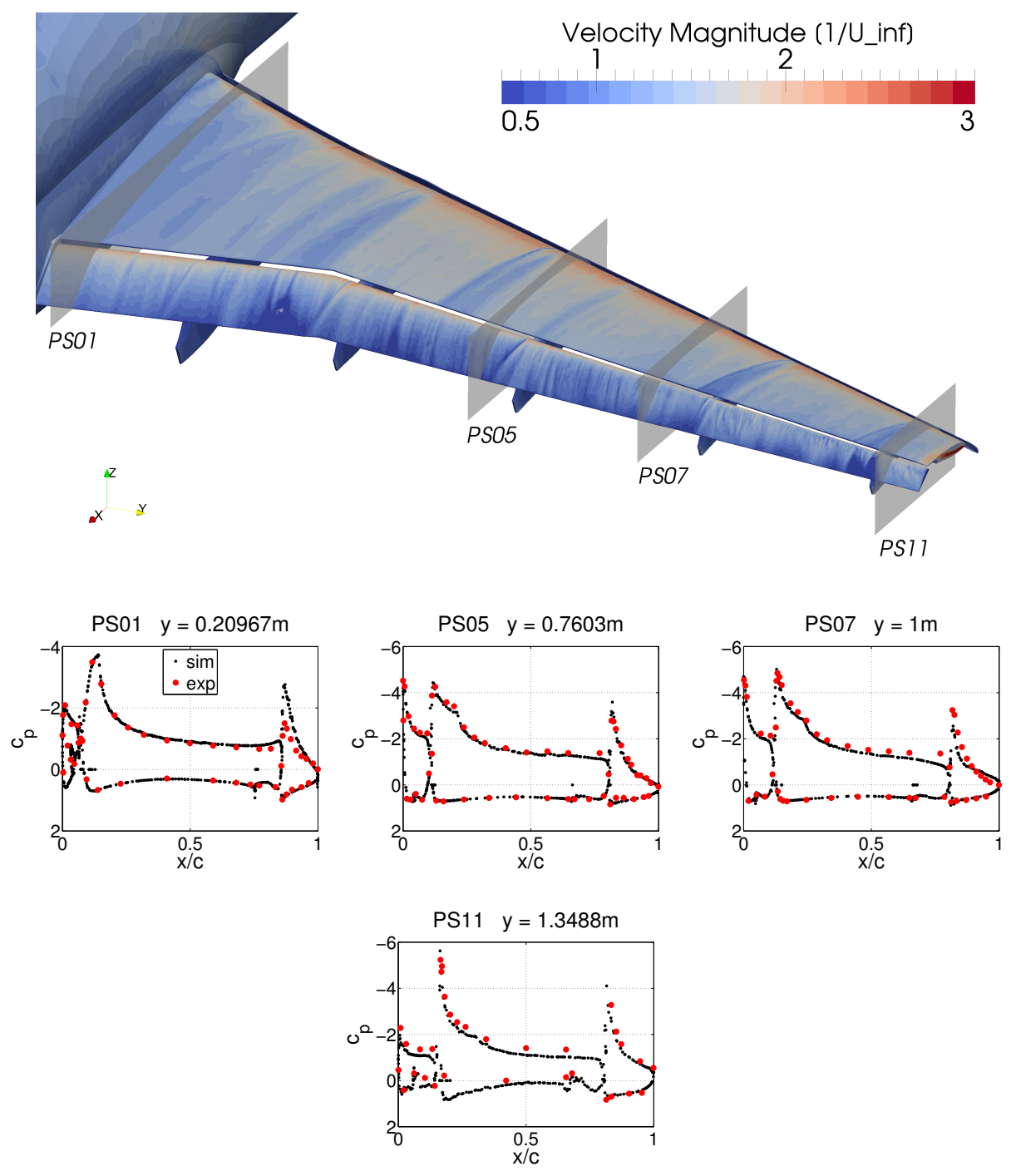

Figure 4. Mean velocity contours and measurement plane locations (upper), and pressure coefficient, $C_{P}$, vs. normalized local chord, $x / c$, (lower) for the angle of attack $\alpha=12^{\circ}$.

at half wing-span, $P S 05$, and two others on the outboard side, closer to the wing tip, $P S 07$ and $P S 11$. In the figure it is also possible to see the location of these measurement stations together with velocity contours on the wing surface (similar to a "limiting streamlines" plot).

The degree of agreement is good on all three elements (slat, fixed wing and flap) on both pressure and suction sides for measurements up to half wing-span. The only difference is a slightly steeper pressure gradient towards the trailing edge of the fixed wing in the simulation at half wing-span and also a stronger suction peak on the flap suction side at the measurement station closest to the aircraft body (PS01). As we approach the wing tip, however, this agreement deteriorates. On the fixed wing, e.g., the simulation result shows consistently lower suction than the measured curve. This should partially explain the lower lift coefficient obtained in the simulation, as seen in Figure 2(a). However, we expect this difference to become less pronounced as the mesh is further refined, at least if the trend indicated in Figure 2(b) and 2(c) holds.

Similar results are shown in Figure 5 for $\alpha=22.4^{\circ}$. A comparison with Figure 4 reveals stronger suction peaks for this angle of attack in both simulation and measurements, which is in accordance with the higher lift obtained for $\alpha=22.4^{\circ}$ in both campaigns. In the examination of Figure 5 alone, we find that the agreement between measured and computed pressure coefficients is excellent at half wing-span on all three wing elements. Moreover, a good match is also seen for the suction peaks on the slat at the inboard measurement section (PS01) and on the main wing at the outboard measurement section (PS11). Apart 

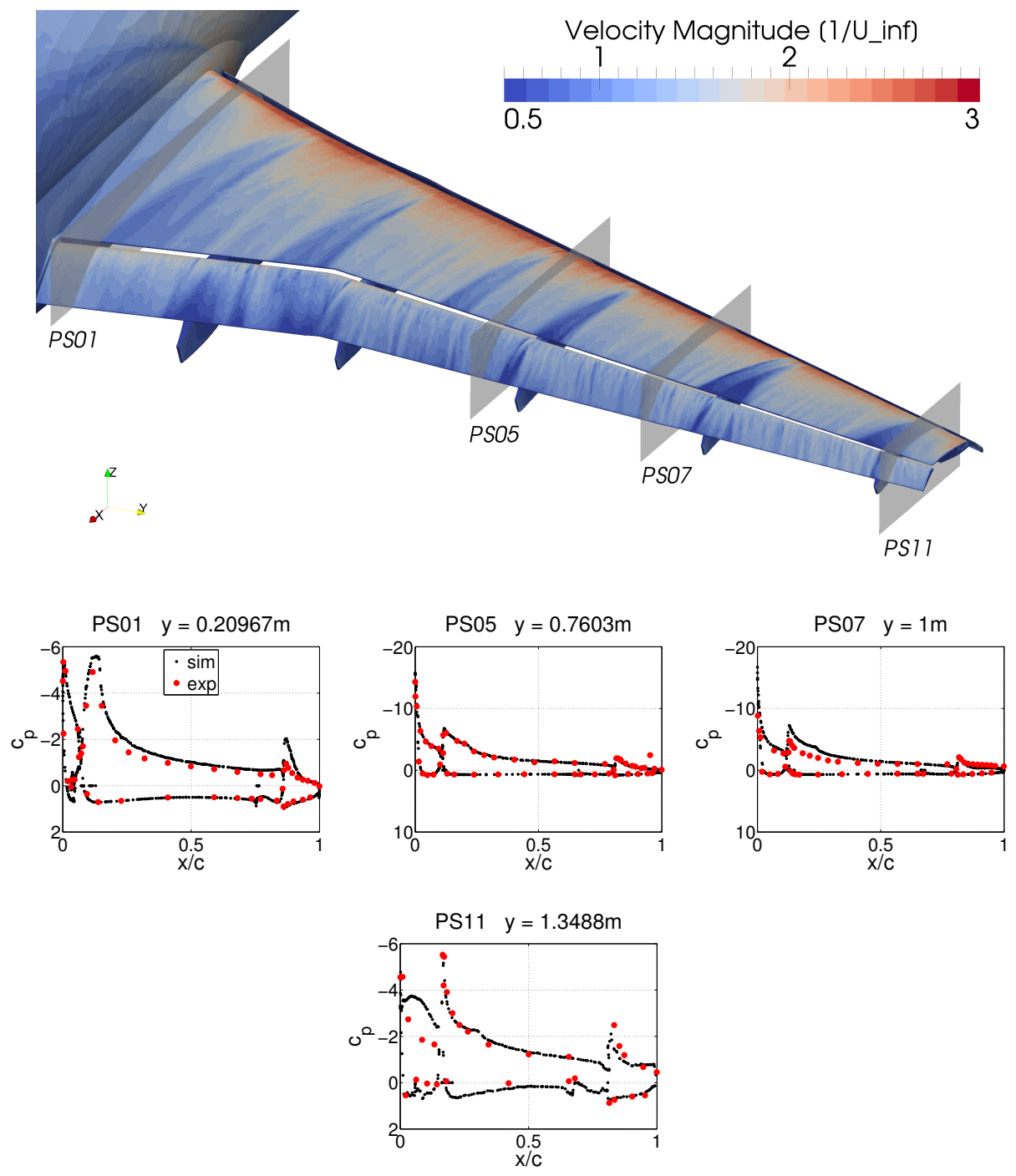

Figure 5. Mean velocity contours and measurement plane locations (upper), and pressure coefficient, $C_{P}$, vs. normalized local chord, $x / c$, (lower) for the angle of attack $\alpha=22.4^{\circ}$.

from these encouraging results, poor agreement is seen on the fixed wing at sections PS01 and PS07, where the simulation curves show a more accentuated suction, which should explain the overshoot of lift coefficient seen in Figure 2(d), Also poor is the agreement on the slat at section PS11.

\section{Flow Visualization}

The velocity contours shown in Figures 4 and 5 reveal some common features that are intrinsically related to the geometry of the DLR-F11 aircraft. A pattern of low velocity streaks, alternating with areas of somewhat higher velocity, is seen on the suction side of the fixed wing for both angles of attack. This is a caused by separation at the slat tracks upstream, which are better visualized in Figure 1 .

In order to illustrate the unsteady, turbulent content of our simulations, Figure 6 shows a snapshot in time of a volume rendering of the $\lambda_{2}$ vorticity criterion for the angle of attack $\alpha=12^{\circ}$. In the figure, we clearly see wing-tip vortices emanating from both wings. 


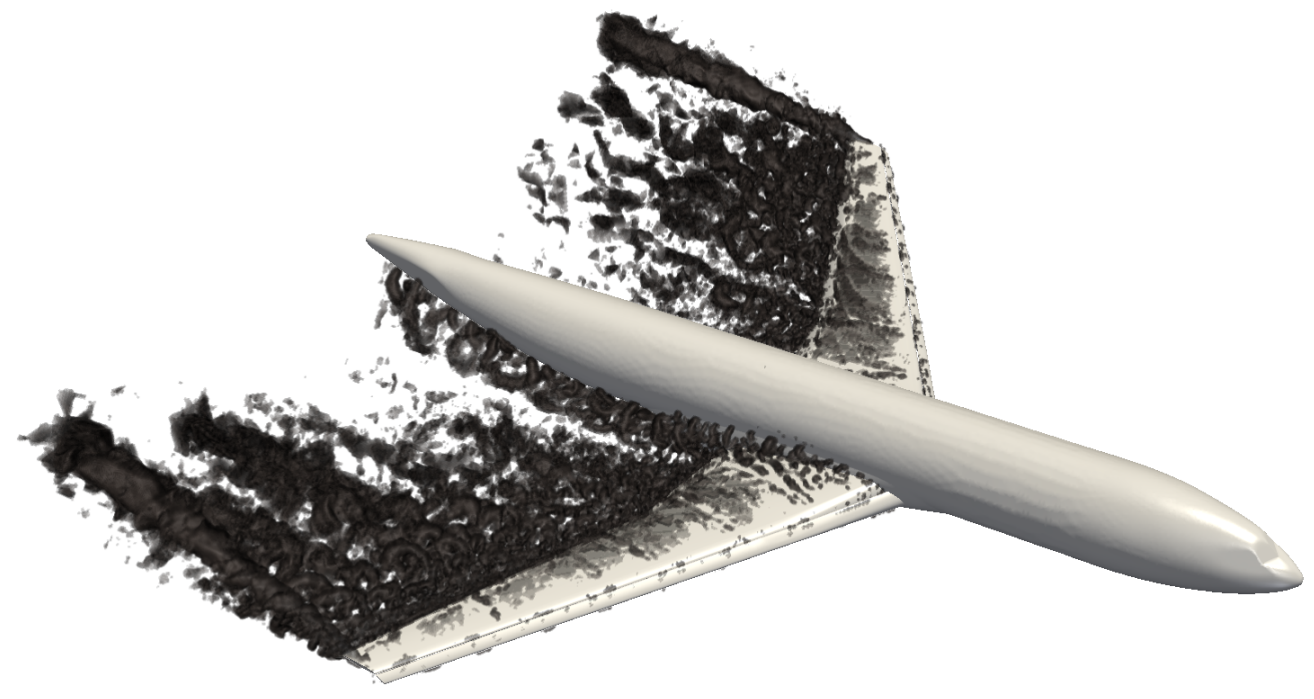

Figure 6. Volume rendering of $\lambda_{2}$ vorticity criterion for the angle of attack $\alpha=12^{\circ}$, snapshot in time.

\section{Preliminary results for "config 5"}

We are currently working on a set of simulations for future publications, where we intend to compare results obtained with "config 4" against results obtained with "config 5". These two configurations differ somewhat, the latter being slightly closer to the experimental model due to the presence of pressure tube bundles near the slat tracks.

Figures 7(a) and 7(b) show a comparison of the results obtained with "config 4" and "config 5 " for the angles of attack $\alpha=22.4^{\circ}, 24^{\circ}$. The computed values obtained for $C_{L}$ and $C_{D}$ with "config 5 " (the red curves) indicate that the flow is indeed stalled: we have a clear lift break when moving from $\alpha=22.4^{\circ}$ to $24^{\circ}$, which is consistently followed by an increase in drag force. For "config 4", a similar drop in the lift curve observed; the drag coefficient, however, does not increase to the levels observed in the experiments, which indicates that simulations performed with "config 4" are not suitable for direct comparisons with measurements near stall. Figures $7(\mathrm{c})] 7(\mathrm{f})]$ show $C_{L}$ and $C_{D}$ as a function of the number of mesh cells for the both angles and both "config's". The trends observed for $C_{L}$ and $C_{D}$ as the mesh is refined reinforce the results illustrated in Figures $7(\mathrm{a})$ and $7(\mathrm{~b})$.

Next, we compare the stalled angle, $\alpha=24^{\circ}$, with the non-stalled angle, $\alpha=22.4^{\circ}$, for "config 5 ", by a volume rendering of the magnitude of the velocity at a snapshot in time in Figure 8 . The velocity values in the range $U=[0.6,1.4]$ have zero opacity to reveal the high velocity in red on the leading edges of the wing components and the low velocity in blue in the wake. We clearly see a separated wake region appearing around the trailing edge of the flap for $\alpha=22.4^{\circ}$, but covering almost the entire surface of the main wing and flap for the stall case at $\alpha=24^{\circ}$ (except close to the aicraft body, where no clear wake region is seen).

\section{Conclusions}

This paper contains the results obtained with the G2 method for the HiLiftPW-2 held in San Diego, California, in 2013. G2 is a time-resolved FEM for turbulent flows with no turbulence modeling, and with an automatic mesh generation algorithm based on an a posteriori error estimate. These features of G2 characterize it as a parameter-free method, where no a priori knowledge of the flow is needed during the problem formulation stage, nor during the mesh generation process. Moreover, in G2, turbulent boundary layers are modeled by a slip with friction boundary condition, and thus no boundary layer mesh is needed. 
We believe the results presented in this report are "very promising". Although no definite convergence is shown in Figures 2 and 3 , our results approach the experimental values as the mesh is refined. Moreover, we were able to predict stall for "config 5" at similar angles of attack as in the experiments, which is a significant physical outcome of our simulations.

\section{Acknowledgments}

The authors would like to acknowledge the financial support from the Swedish Foundation for Strategic Research, the European Research Council and the Swedish Research Council. The simulations were performed on resources provided by the Swedish National Infrastructure for Computing (SNIC) at PDC - Center for High-Performance Computing and on resources provided by the "Red Española de Supercomputación" and the "Barcelona Supercomputing Center - Centro Nacional de Supercomputación".

The initial volume mesh was generated with ANSA from Beta-CAE Systems S. A., who generously provided an academic license for this project.

\section{References}

${ }^{1}$ Rumsey, C., " $2^{\text {nd }}$ AIAA CFD High Lift Prediction Workshop (HiLiftPW-2) (http://hiliftpw.larc.nasa.gov/)," 2013.

${ }^{2}$ Choudhari, M. and Visbal, M., "Second Workshop on Benchmark problems for Airframe Noise Computations (BANCII) (https://info.aiaa.org/tac/ASG/FDTC/DG/BECAN_files_/BANCII.htm)," 2012.

${ }^{3}$ Hoffman, J. and Johnson, C., Computational Turbulent Incompressible Flow, Vol. 4 of Applied Mathematics: Body and Soul, Springer, 2007.

${ }^{4}$ Hoffman, J., "Computation of mean drag for bluff body problems using Adaptive DNS/LES," SIAM J. Sci. Comput., Vol. 27(1), 2005, pp. 184-207.

${ }^{5}$ Hoffman, J., "Adaptive simulation of the turbulent flow past a sphere," J. Fluid Mech., Vol. 568, 2006, pp. 77-88.

${ }^{6}$ Hoffman, J. and Johnson, C., "A new approach to Computational Turbulence Modeling," Comput. Methods Appl. Mech. Engrg., Vol. 195, 2006, pp. 2865-2880.

${ }^{7}$ Hoffman, J., "Efficient computation of mean drag for the subcritical flow past a circular cylinder using General Galerkin G2," Int. J. Numer. Meth. Fluids, Vol. 59(11), 2009, pp. 1241-1258.

${ }^{8}$ Houzeaux, G., Vázquez, M., Aubry, R., and Cela, J., "A massively parallel fractional step solver for incompressible flows," Journal of Computational Physics, Vol. 228, No. 17, 2009, pp. 6316-6332.

${ }^{9}$ Hoffman, J. and Jansson, N., A computational study of turbulent flow separation for a circular cylinder using skin friction boundary conditions, Ercoftac, series Vol.16, Springer, 2010.

${ }^{10}$ Hoffman, J. and Johnson, C., "Resolution of d'Alembert's Paradox," J. Math. Fluid Mech., Published Online First at www.springerlink.com: 10 December 2008.

${ }^{11}$ Vilela de Abreu, R., Jansson, N., and Hoffman, J., "Adaptive computation of aeroacoustic sources for a rudimentary landing gear," Int. J. Numer. Meth. Fluids, Vol. 74, No. 6, 2014, pp. 406-421. 


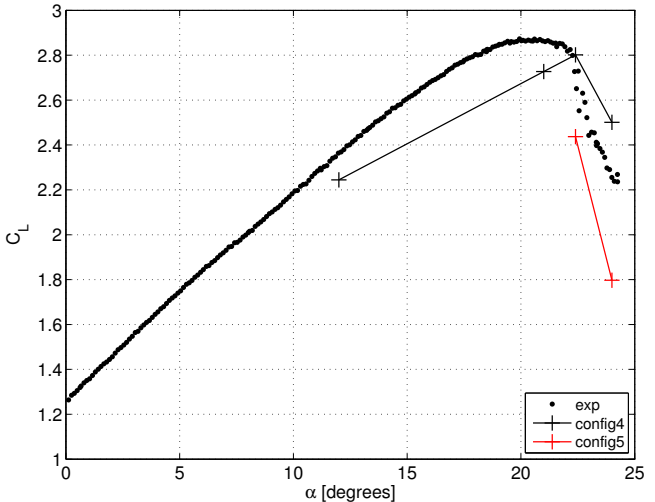

(a) Lift vs. $\alpha$, finest mesh available.

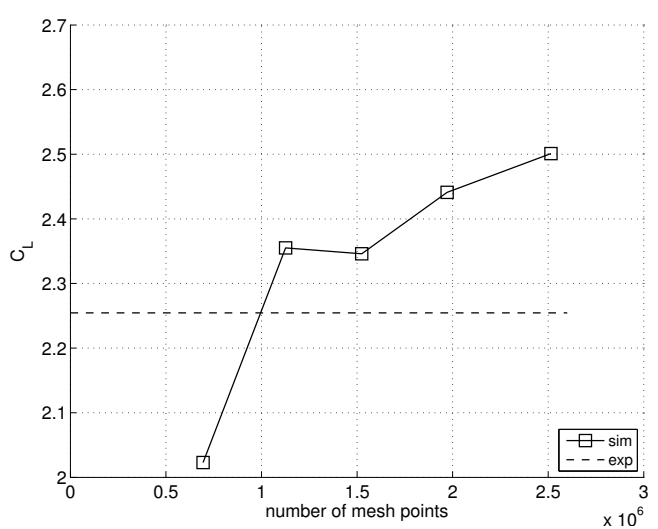

(c) Lift vs. number of mesh points, $\alpha=24^{\circ}$, "config 4".

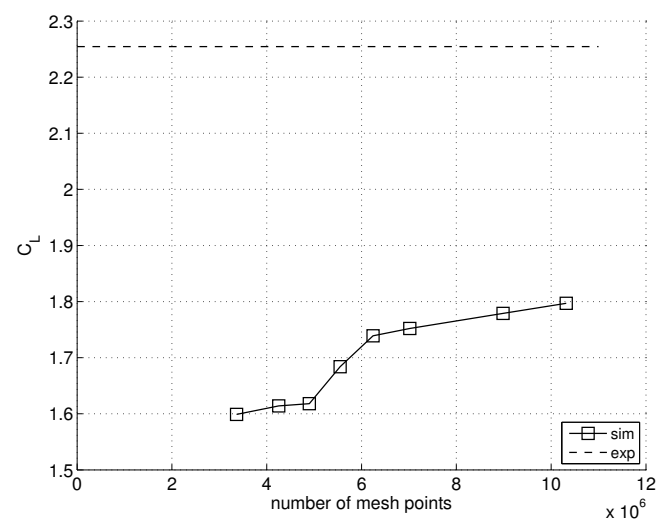

(e) Lift vs. number of mesh points, fig $5 "$.

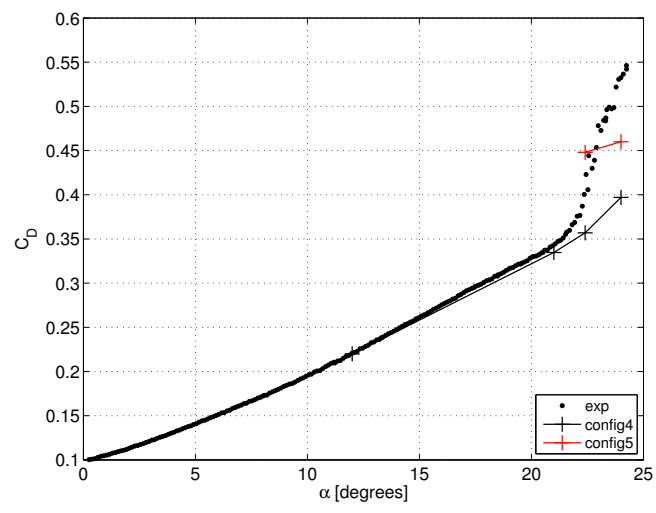

(b) Drag vs. $\alpha$, finest mesh available.

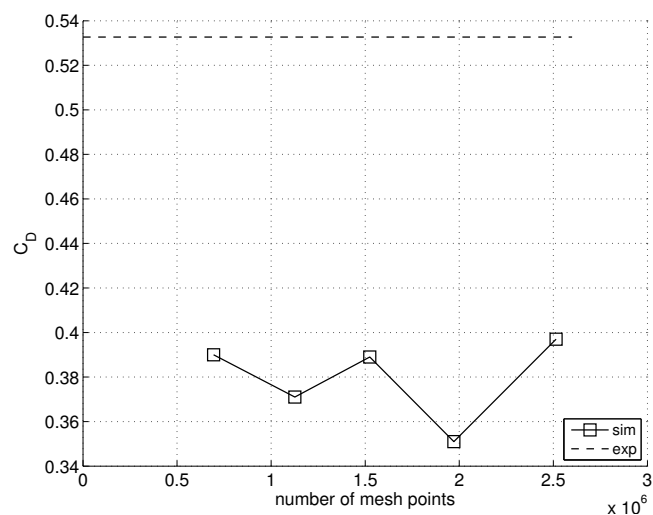

(d) Drag vs. number of mesh points, $\alpha=24^{\circ}$, "config 4".

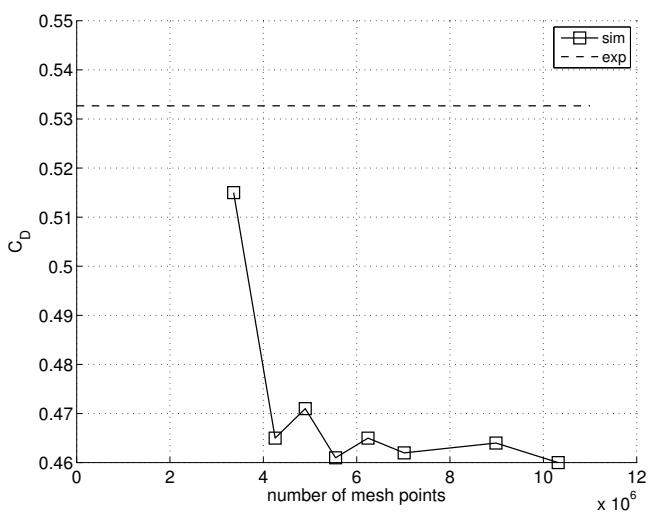

(f) Drag vs. number of mesh points, $\alpha=24^{\circ}$, "config 5".

Figure 7. Lift and drag coefficients, $C_{L}$ and $C_{D}$, vs. angle of attack, $\alpha$, and vs. number of mesh points, a comparison of the results for "config 4" and "config 5". 

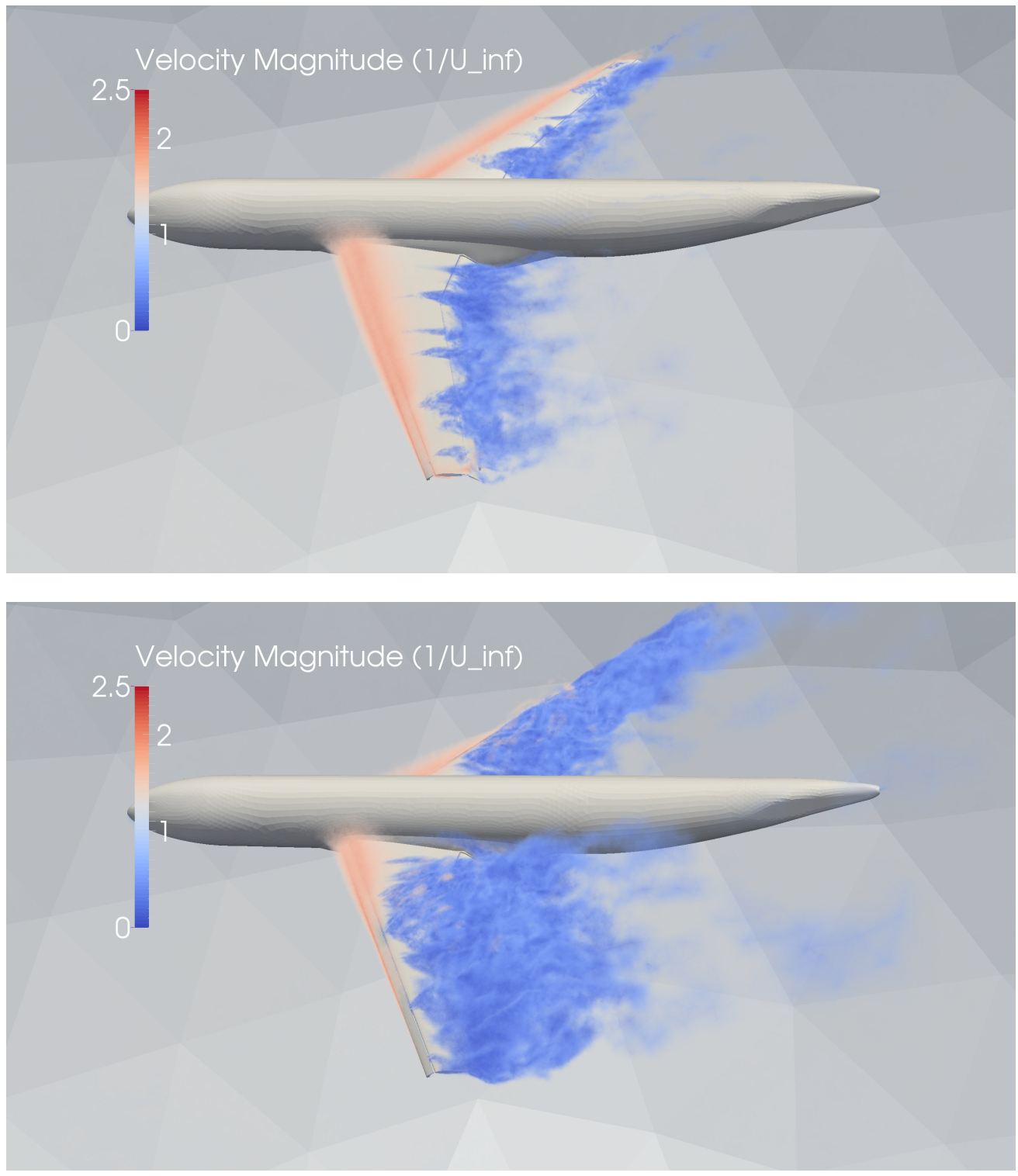

Figure 8. Volume rendering of a snapshot in time of the magnitude of the velocity for the angle of attack $\alpha=22.4^{\circ}$ (upper) and $\alpha=24^{\circ}$ (lower) illustrating stall. The transfer function for the volume rendering has zero opacity in the range $U=[0.6,1.4]$ with full opacity at the endpoints $U=0$ and $U=2.5$ of the color scale. 